BIOKEMISTRI 17(2):83-94 (Dec 2005)

Available online at http://www.bioline.org.br/bk. Abstracted online at www.ajol.info/journals/biokem

Printed in Nigeria

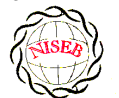

OVigerian Society for O̊xperimental Siology

\title{
Uncoupling proteins 2 and 3 alter mitochondrial membrane phospholipid fatty acyl composition in yeast
}

\section{Augustine OCLOO}

\author{
Medical Research Council Dunn Human Nutrition Unit, Hills Road, Cambridge CB2 2XY, \\ United Kingdom
}

Received 23 September 2005

MS/No BKM/2005/041, @ 2005 Nigerian Society for Experimental Biology. All rights reserved.

\begin{abstract}
Mouse UCP1, human UCP2 and UCP3 were expressed in yeast (Saccharomyces cerevisiae). Total phospholipids were extracted and their fatty acyl composition was analysed. Expression of hUCP2 and hUCP3 significantly altered the mitochondrial phospholipid fatty acyl composition. Palmitoleoyl groups (16:1(n-7)) decreased while oleoyl groups (18:1(n-9)) increased, leading to a significant increase in the 18:1(n-9)/16:1(n-7) ratio and in the average chain length. Expression of mUCP1 did not cause any significant changes in fatty acyl composition
\end{abstract}

Key words: Yeast, Saccharomyces cerevisiae, phospholipid, fatty acyl group, uncoupling protein, adenine nucleotide translocase, desaturase.

\footnotetext{
Abbreviations used: ACL, average chain length; MUFA, monounsaturated fatty acids; PUFA, polyunsaturated fatty acids; SFA, saturated fatty acids; UCP, uncoupling protein; UI, unsaturation index; UFA, unsaturated fatty acids
}

E-mail: ao@ mrc-dunn.cam.ac.uk or augustineocloo@yahoo.co.uk; Tel: (+44) 1223503350 


\section{INTRODUCTION}

Adenosine triphosphate (ATP) is the chemical energy currency in living systems. In the 1940's mitochondria were discovered to be the main site of oxidative ATP synthesis in eukaryotes ${ }^{1}$. In 1961, Peter Mitchell proposed that a proton motive force generated across the mitochondrial membrane drives ATP synthesis ${ }^{2}$ (the proton motive force is coupled to ATP synthesis). In other words, ATP synthesis occurs when protons that are pumped out of the mitochondrial matrix, during transfer of electrons along the electron transport system, return through the ATP synthetase.

It was first observed in the early $1970 \mathrm{~s}^{3,4}$ and confirmed in the early $1990 \mathrm{~s}^{5}$ that not all the protons that go out of the mitochondrial matrix return through the ATP synthetase, a significant amount return through other pathways and are thus not available for ATP synthesis. This process is termed mitochondrial uncoupling or proton leak. Mitochondrial uncoupling or proton leak have been implicated in thermogenesis ${ }^{6}$, diabetes $^{7,8}$, obesity ${ }^{9}$, ageing ${ }^{10}$ and cachexia ${ }^{11}$.

Thermogenin or Uncoupling protein 1 (UCP1) uncouples mitochondrial ATP synthesis from substrate oxidation in mammalian brown adipose tissue ${ }^{12,13}$. It catalyzes an inducible leak of protons through the mitochondrial inner membrane that is sensitive to purine nucleotides like ATP and GDP. When activated by fatty acids, UCP1 short-circuits the electrochemical proton gradient and leads to the production of heat (thermogenesis) ${ }^{6,14}$. In addition to the inducible proton leak catalysed by UCP1, is a basal proton leak present in the mitochondrial of all organisms. The mechanism of this basal leak is unresolved. Among the several hypothesis that have been proposed are that the basal proton leak is catalysed by specific proteins, is due to interaction between membrane lipids and proteins and is conductance by the phospholipid bilayer.

Supporting the phospholipid bilayer hypothesis are consistent correlations between the basal proton leak and various phospholipid fatty acyl parameters in that conditions in which the basal proton leak changes, the phospholipids fatty acid composition also changes in specific manner. In general, proton leak correlates consistently with the mitochondrial membrane phospholipid fatty acid composition across a range of species $^{15}$. In all, 13 out of 28 phospholipid fatty acyl group parameters studied show very good correlations with proton leak. The mitochondrial membrane phospholipids 20:3(n-6), 20:4(n-6), 20:6(n-3) contents, UI and 20:4(n-6)/18:2(n-6) ratio correlates positively with proton leak while 14:0, 16:1(n-7), 18:1(n9), 18:3(n-6), 18:3(n-3) contents, percent $n-9$ fatty acids and percent monounsaturates correlate negatively ${ }^{15-20}$.

Transgenic overexpression of human UCP3 (hUCP3) in mouse skeletal muscle mitochondria leads to animals that are hyperphagic and lean ${ }^{21}$. The proton conductance of skeletal muscle mitochondria isolated from these mice is increased and satisfactorily explains the phenotype ${ }^{22}$. Intriguingly, the phospholipid fatty acyl composition of skeletal muscle mitochondria isolated from mice overexpressing hUCP3 was significantly different from that of mitochondria from wild type mice ${ }^{23}$. There were higher amounts of 18:0 and 22:6(n-3) and lower amounts of 16:0, $18: 1(n-9)$ and 18:2(n-6) in UCP3overexpressers. These changes were similar to those previously found to correlate across a range of species and treatments with basal proton conductance ${ }^{15,19,20}$, raising the possibility that the increase in proton conductance in mitochondria from these mice (and maybe also from the yeast expressing hUCP2 and hUCP ${ }^{24-}$ ${ }^{26}$ might be caused indirectly by changes in mitochondrial phospholipid fatty acyl composition. Conversely, the changes in fatty acyl group composition in the transgenic mice might be (i) a response to increased food consumption; (ii) a response to high levels of membrane protein expression; (iii) a response to the uncoupling effect of hUCP3 or (iv) a more direct effect caused by improperly folded protein.

In the present paper, it is shown that expression of hUCP2 and hUCP3 in yeast significantly alters phospholipid fatty acyl composition. I eliminate explanations (i) to (iii) and suggest that the change is caused by (iv), a direct effect of improperly folded protein. 


\section{EXPERIMENTAL Yeast}

Saccharomyces cerevisiae diploid W303

$(\mathrm{a} / \alpha)$ cells expressing the mammalian uncoupling proteins 1,2 , and 3 or containing their respective empty vectors were obtained from previous stocks ${ }^{24-26}$. Yeast cells expressing modest levels of mouse UCP1 (pBF307) was made by inserting a BamHI-EcoRI PCR fragment, containing the mouse UCP1 coding sequence preceded at the $5^{\prime}$ end by GCC, into the BamHI-EcoRI site in the multiple cloning site in pYES2 from Invitrogen ${ }^{25}$. The pYES2 empty vector contains no gene in its multiple cloning site. Yeast cells expressing nonphysiological levels of mouse UCP1 (pBF352), human UCP2 or UCP3 (pBF353 or pBF354 respectively) was constructed by using mouse UCP1, human UCP2 or human UCP3 cDNA respectively as a template for PCR-amplification with the primers

5'-GGATCCGGATCCATAATGGTTGGGTTCAAGGC-3' and

3'-AGCAGGGGATCCTCAGAAGGGAGCCTCTC-5' 24 . The product was digested with BamHI and inserted into pKV49 that had been restricted with $B g l I I$. The pKV49 empty vector (pKVev) contains no gene in its multiple cloning site.

Yeast cells were grown as described previously ${ }^{24,26}$. Yeast cells from glycerol stocks were grown on solid medium and were incubated at $30^{\circ} \mathrm{C}$ for 48 hours. A single cell colony was obtained and inoculated on a fresh solid medium and incubated at $30^{\circ} \mathrm{C}$ for 48 hours. These cells were then used for the liquid cultures. Different mutants were grown on slightly different media as required for selectivity. Precultures of cells transformed with pYES2-based vectors (pYES2 empty vector control or pBF307, which expresses a modest level of mouse UCP1) were grown in selective lactate medium containing $2 \% \mathrm{w} / \mathrm{v}$ Lactic Acid, $0.67 \%$ w/v YNB, $0.1 \%$ w/v $\mathrm{KH}_{2} \mathrm{PO}_{4}, 0.12 \%$ w/v $\left(\mathrm{NH}_{4}\right)_{2} \mathrm{SO}_{4}, 0.1 \%$ Glucose, $0.1 \%$ w/v Casamino acids (from Difco); the main cultures were grown in $2 \%$ Galactose medium containing $0.67 \% \mathrm{w} / \mathrm{v}$ YNB, $2 \% \mathrm{w} / \mathrm{v}$ Galactose, $0.1 \% \mathrm{w} / \mathrm{v}$ Casamino acid, Tryptophan $(20 \mathrm{mg} / \mathrm{ml})$, Adenine $(40 \mathrm{mg} / \mathrm{ml})^{26}$. Precultures of cells transformed with the pKV49-based vectors (pKV empty vector control (pKVev), pBF352, pBF353 or pBF354 ${ }^{24-26}$, expressing high levels of mouse UCP1, human UCP2 and human $\mathrm{UCP} 3$ respectively) were grown in $2 \%$ selective lactate medium containing 1X Amino Acid drop out stock (leucine drop out) in place of casamino acid; the main cultures were grown in $3 \%$ selective lactate medium containing $3 \% \mathrm{w} / \mathrm{v}$ Lactic Acid in place of $2 \% \mathrm{w} / \mathrm{v}$ Lactic Acid and 1X Amino Acid drop out stock (leucine drop out) in place of casamino acid. The main cultures were induced for $4 \mathrm{~h}$ with $1 \%(\mathrm{w} / \mathrm{w})$ galactose as described previously ${ }^{24-26}$.

Yeast growth was measured as follows: precultures of $\mathrm{pKVev}$ and $\mathrm{pBF} 354$ were grown overnight as described previously ${ }^{24}$. They were diluted in triplicate to an optical density at 600 $\mathrm{nm}\left(\mathrm{OD}_{600}\right)$ of 0.2 into $3 \%$ selective lactate medium containing $1 \%(\mathrm{w} / \mathrm{v})$ galactose and the indicated concentrations of dinitrophenol, then $\mathrm{OD}_{600}$ was measured over $12 \mathrm{~h}$.

\section{Phospholipids}

Yeast mitochondria were isolated by centrifugation as described previously ${ }^{27}$. Mitochondrial protein concentration was determined by biuret assay. The presence of UCP was determined by western blot analysis as described previously ${ }^{24}$.

Total mitochondrial lipids were extracted as previously described ${ }^{28}$. About $2 \mathrm{mg}$ mitochondrial protein was extracted in $10 \mathrm{ml}$ of $2: 1 \mathrm{v} / \mathrm{v}$ chloroform-methanol containing $0.05 \%$ w/v butylated hydroxytoluene. Lipids were redissolved in 1-2 $\mathrm{ml}$ of chloroform. Phospholipids were isolated by silicic acid chromatography by using silica cartridges ${ }^{17}$. Briefly, the total lipid extract was loaded on a silicic acid column. The column was washed with $2 \mathrm{ml}$ of chloroform to elute neutral lipids. The phospholipids were eluted with $2 \mathrm{ml}$ of methanol into a round-bottom flask. The mixture was dried under nitrogen at $25^{\circ} \mathrm{C}$ and dissolved in 19/1 (v/v) chloroform:methanol.

\section{Fatty acid analysis}

Fatty acid methyl esters were prepared in the presence of boron trifluoride in methanol as previously described ${ }^{28}$. They were purified on a florisil column, dried under nitrogen, re- 
dissolved in n-hexane and analysed on a gas chromatograph. Fatty acid methyl esters were detected using a flame ionisation detector and identified against fatty acid methyl ester standards. Amounts were measured by integration of peak areas after baseline correction and mol\% of each fatty acid was calculated as (100 x mole fa)/(sum of mole fa for all fa). No unidentified peak contributed more than $0.1 \%$ of the total fatty acid methyl esters present. Unsaturation index (UI) was calculated as $[(\Sigma \mathrm{mol} \%$ monoenoic $)+(2 \times \Sigma \mathrm{mol} \%$ dienoic $)$ $+(3 \mathrm{x} \Sigma \mathrm{mol} \%$ trienoic $)+(4 \mathrm{x} \Sigma \mathrm{mol} \%$ tetraenoic $)+(5 \times \Sigma m o l \%$ pentaenoic $)+(6 \mathrm{x}$ $\Sigma \mathrm{mol} \%$ hexaenoic)].

\section{Statistical analysis}

Student's t-test was used for statistical calculations.

\section{RESULTS}

\section{Effect of expression of uncoupling proteins on mitochondrial phospholipid fatty acyl composition}

The starting point for the experiments in the present paper was the observation that skeletal muscle mitochondria from mice transgenically overexpressing human UCP3 had altered phospholipid fatty acyl composition compared to wild type controls ${ }^{23}$. Mitochondria from UCP3 overexpressers had less 16:0, 18:1(n-9) and 18:2(n-6), and more 18:0 and $22: 6(n-3)$, leading to lower total monounsaturates and higher total n-3 polyunsaturates, average chain length, unsaturation index and peroxidizability index.

These fatty acyl composition differences were reminiscent of those previously found to correlate with basal proton conductance in mitochondria from a range of species and treatments ${ }^{15,19,20}$, suggesting that high protein expression, or some secondary result of uncoupling, may cause the observed correlation between basal proton conductance and phospholipid fatty acyl composition. There are some differences between studies, but over a wide range of different vertebrate species and experimental treatments there is a positive correlation of basal proton conductance with n-3 polyunsaturate content, particularly 22:6(n-3), and a negative correlation with monounsaturate content, particularly $18: 1(n-9)^{15,16,19,20,30,31]}$. To explore whether expression of UCPs was directly responsible for the altered phospholipid fatty acyl profile in mouse skeletal muscle mitochondria, we examined the effects of expressing mammalian UCPs on phospholipid fatty acyl composition in yeast. Figure 1 shows the result of western blot analysis of mitochondria from yeast expressing mouse UCP1 and yeast containing the empty vector. There is no detectable amount of UCP in yeast mitochondria. Accordingly, there were bands at every concentration of mitochondrial protein (10 $\mu \mathrm{g}, 20 \mu \mathrm{g}$ and $30 \mu \mathrm{g}$ ) in mitochondria from yeast expressing high levels of mouse UCP1 (pBF352), and no bands in the empty vector.

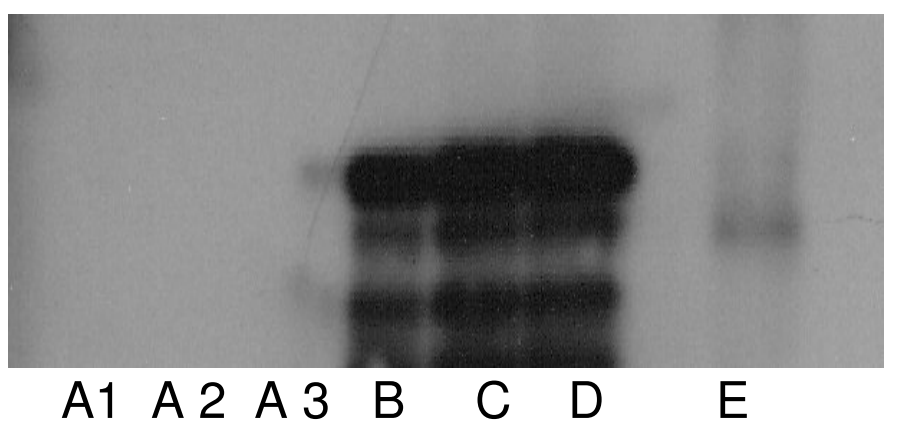

Fig. 1: Expression of mouse UCP1 in yeast. Western blot analysis of expression of UCP1 in mitochondria from yeast expressing high levels of mouse UCP1 (pBF352) compared to empty vector control (pKVev). Wells A1, A2 and A3 contains $10 \mu \mathrm{g}, 30 \mu \mathrm{g}$ and $50 \mu \mathrm{g}$ mitochondrial protein respectively of mitochondria from yeast expressing the empty vector, B, C and D contains $10 \mu \mathrm{g}, 30 \mu \mathrm{g}$ and $50 \mu \mathrm{g}$ mitochondrial protein respectively of mitochondria from yeast expressing high levels of mouse UCP1, E represents pure brown adipose tissue UCP1.

The phospholipid fatty acyl composition of yeast mitochondria in the present study differed from that of mammals, but was comparable to previous yeast results ${ }^{29}$. In yeast mitochondria, more than $80 \%$ of the total fatty acyl groups are either palmitoleoyl [16:1(n-7)] or oleoyl [18:1(n-9)] (Fig 2).

The expression of hUCP3 at high levels in yeast using the $\mathrm{pKV}$ vector $^{24}$ causes uncoupling that is an artifact of overexpression ${ }^{24}$. It was observed that such overexpression of hUCP3 resulted in small but significant changes in the mitochondrial phospholipid fatty acyl profile compared to yeast transformed with the pKV49 empty vector control (pKVev) (Fig 2a). 


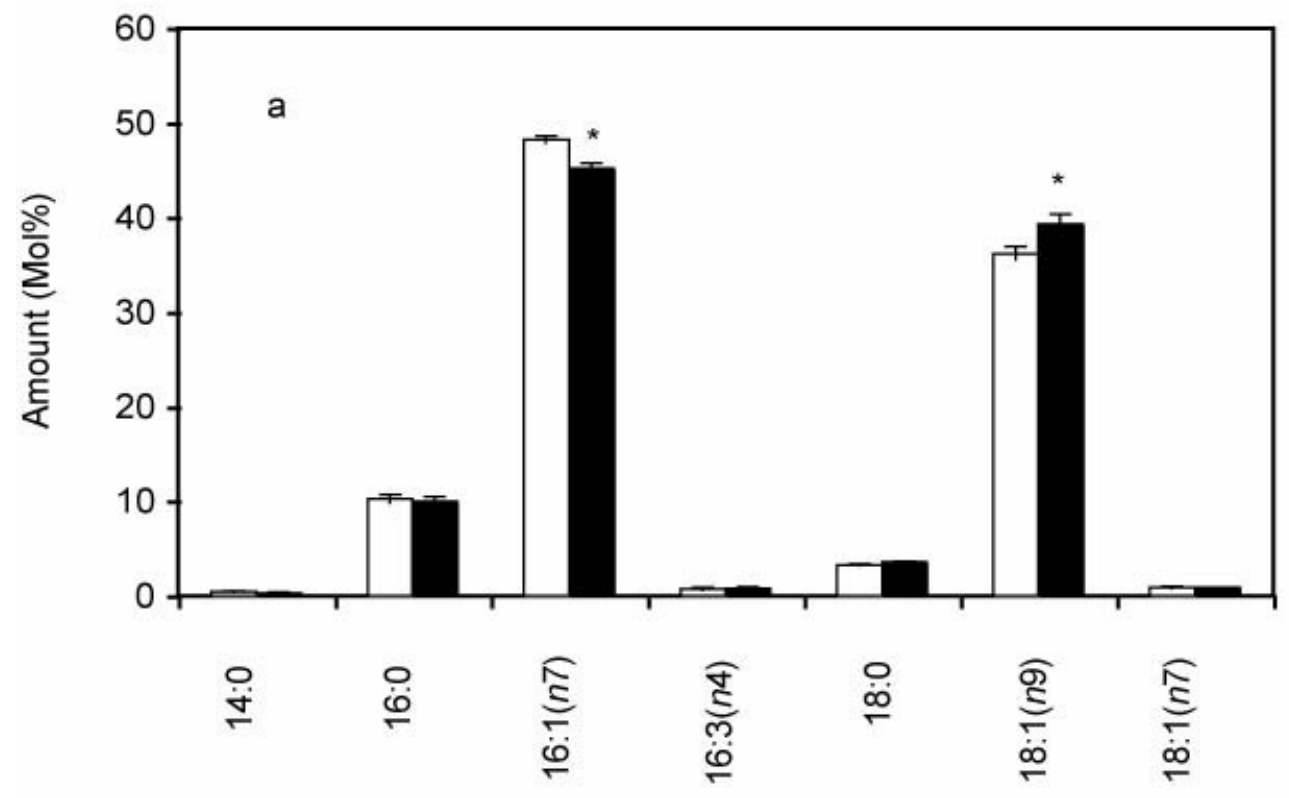

Fatty acyl group

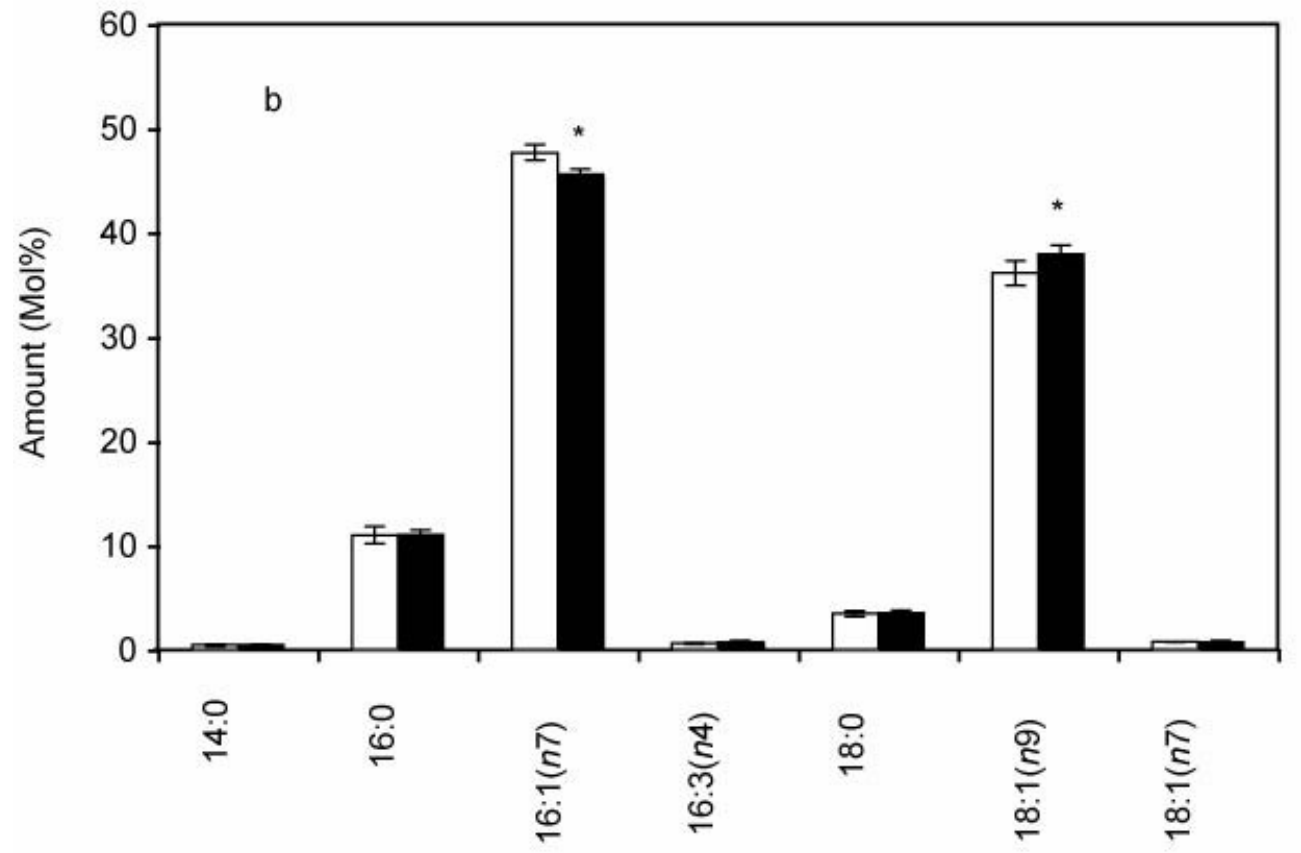

Fatty acyl group

Fig. 2. Total mitochondrial phospholipid fatty acyl profile of yeast mitochondria expressing hUCP3 or hUCP2

(a) Mitochondria from yeast expressing hUCP3 (pBF354) (black bars) compared to paired empty vector control (pKVev) (white bars). Values are means \pm SEM of 4 independent experiments. ${ }^{*} p<0.05$ compared to pKVev; C16:1(n-7), $p=0.003 ; \mathrm{C} 18: 1(n-9), p=0.01$. (b) Mitochondria from yeast expressing hUCP2 (pBF353) (black bars) compared to paired empty vector control (pKVev) (white bars). Values are means \pm SEM of 5 independent experiments. ${ }^{*} p<0.05$ compared to $\mathrm{pKVev}$ : C16:1(n-7), $p=0.02 ; \mathrm{C} 18: 1(n-9), p=0.04$ 
Table I: Global fatty acyl parameters of mitochondrial phospholipids from hUCP3-expressing yeast compared to paired empty vector (pKVev).

\begin{tabular}{llll}
\hline Index & hUCP3 & pKVev & $p$ value \\
\hline SFA $(\mathrm{mol} \%)$ & $13.9 \pm 0.5$ & $13.4 \pm 0.5$ & 0.84 \\
UFA $(\mathrm{mol} \%)$ & $86.1 \pm 0.5$ & $86.2 \pm 0.5$ & 0.84 \\
MUFA $(\mathrm{mol} \%)$ & $85.4 \pm 0.5$ & $85.4 \pm 0.5$ & 0.99 \\
ACL & $16.80 \pm 0.02$ & $16.86 \pm 0.02$ & $0.005^{*}$ \\
UI & $87.6 \pm 0.6$ & $87.8 \pm 0.8$ & 0.60 \\
$18: 1(n-9) / 16: 1(n-7)$ & $0.75 \pm 0.02$ & $0.87 \pm 0.03$ & $0.002^{*}$ \\
$18: 1(n-9) / 18: 0$ & $11.2 \pm 0.3$ & $11.0 \pm 0.3$ & 0.78 \\
UFA/SFA & $6.2 \pm 0.2$ & $6.3 \pm 0.3$ & 0.80 \\
$16: 1(n-7) / 16: 0$ & $4.8 \pm 0.2$ & $4.6 \pm 0.2$ & 0.14 \\
$18: 0 / 16: 0$ & $0.32 \pm 0.02$ & $0.36 \pm 0.02$ & $0.004^{*}$ \\
$18: 1(n-7) / 16: 1(n-7)$ & $0.02 \pm 0.00$ & $0.02 \pm 0.00$ & 0.30 \\
Values are means \pm SEM of 4 independent experiments. ${ }^{*} p<0.05$ compared to pKVev control
\end{tabular}

Table II: Global fatty acyl parameters of mitochondrial phospholipids from hUCP2-expressing yeast compared to paired empty vector (pKVev)

\begin{tabular}{llll}
\hline Index & hUCP2 & pKVev & $p$-value \\
\hline SFA (mol\%) & $14.8 \pm 1.2$ & $15.0 \pm 0.6$ & 0.89 \\
UFA (mol\%) & $85.2 \pm 1.2$ & $85.0 \pm 0.6$ & 0.89 \\
MUFA (mol\%) & $84.6 \pm 1.2$ & $84.3 \pm 0.6$ & 0.78 \\
ACL & $16.8 \pm 0.0$ & $16.9 \pm 0.0$ & $0.04^{*}$ \\
UI & $86.4 \pm 1.1$ & $86.5 \pm 0.5$ & 0.82 \\
$18: 1(n-9) / 16: 1(n-7)$ & $0.76 \pm 0.03$ & $0.83 \pm 0.03$ & $0.01^{*}$ \\
$18: 1(n-9) / 18: 0$ & $11.0 \pm 1.3$ & $11.3 \pm 1.3$ & 0.74 \\
UFA/SFA & $6.0 \pm 0.6$ & $5.8 \pm 0.3$ & 0.62 \\
$16: 1(n-7) / 16: 0$ & $4.5 \pm 0.4$ & $4.2 \pm 0.3$ & 0.89 \\
$18: 0 / 16: 0$ & $0.32 \pm 0.0$ & $0.32 \pm 0.02$ & 0.81 \\
$18: 1(n-7) / 16: 1(n-7)$ & $0.02 \pm 0.0$ & $0.02 \pm 0.0$ & 0.89 \\
& & & \\
\hline
\end{tabular}

Values are means \pm SEM of 5 independent experiments. ${ }^{*} p<0.05$ compared to $p K V e v$. 


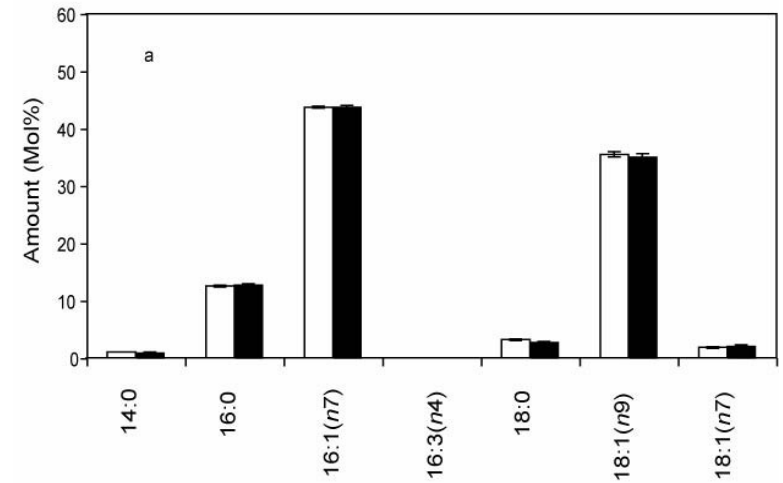

Fatty acyl group

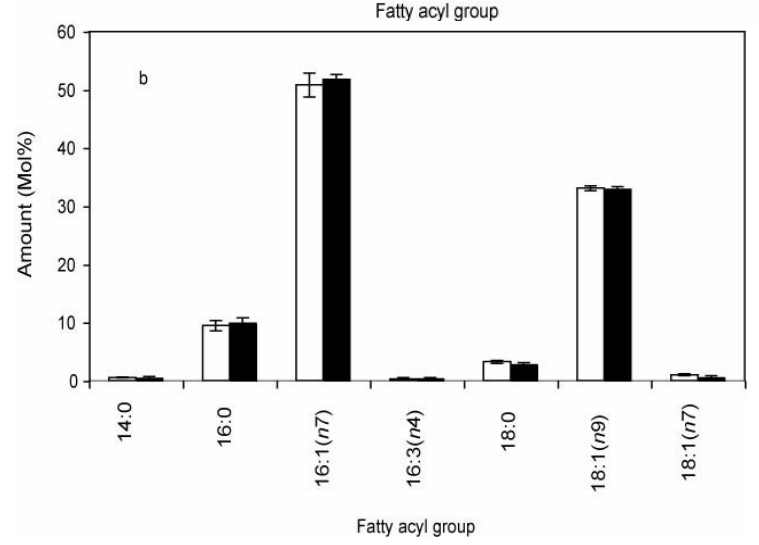

Fig. 3. Total mitochondrial phospholipid fatty acyl profile of yeast mitochondria expressing low or high level of mUCP1.

(a) Mitochondria from yeast expressing a low level of mUCP1 (pBF307) (black bars) compared to paired empty vector control (pYES2) (white bars). Values are means \pm SEM of 7 independent experiments. (b) Mitochondria from yeast expressing a high level of mUCP1 (pBF352) (black bars) compared to paired empty vector control (pKVev) (white bars). Values are means \pm SEM of 3 independent experiments.

In mitochondria from yeast expressing hUCP3, each of the two major fatty acyl species was significantly altered; the level of 16:1(n-7) was decreased to $94 \%$ of control and the level of 18:1(n-9) was increased to $109 \%$ of control. These fatty acyl differences resulted in significant increases in average chain length and 18:1(n-9)/16:1(n-7) ratio in hUCP3 compared to pKVev (Table I).

Expression of hUCP2 in yeast at high levels in yeast using the $\mathrm{pKV}$ vector also causes uncoupling that is an artifact of overexpression $^{26}$. Such overexpression of hUCP2 resulted in the same small but significant changes in the major fatty acyl species as expression of hUCP3 (Fig 2b). In mitochondria from yeast expressing hUCP3, the level of $16: 1(n-7)$ was decreased to $96 \%$ of that in paired empty vector control (pKVev) and the level of 18:1(n-9) was increased to $105 \%$ of control. As they did with UCP3 expression, the average chain length and the $18: 1(n-9) / 16: 1(n-7)$ ratio increased significantly in hUCP2 compared to pKVev (Table II). However, in hUCP3 containing yeast the 18:0/16:0 ratio was significantly higher than in paired empty vector control, whereas in hUCP2 containing yeast this ratio did not change.

In contrast to hUCP2 and hUCP3, modest expression of mUCP1 in yeast leads to native fatty acid-dependent, nucleotide-sensitive UCP1 uncoupling function ${ }^{25}$. Modest expression of mUCP1 using pBF307 had no significant effect on yeast mitochondrial phospholipid fatty acyl composition compared to its pYES2 empty vector control (Fig 3a). High expression of mUCP1 in yeast, however, does cause substantial artifactual uncoupling ${ }^{25}$. Nevertheless, such overexpression of mUCP1 also had no significant effect on yeast mitochondrial phospholipid fatty acyl composition compared to paired empty vector control (pKVev) (Fig 3b).

Effect of chemical uncoupling on mitochondrial phospholipid fatty acyl composition

UCP2 and UCP3 are not properly expressed in yeast mitochondria; they may be partially denatured ${ }^{32}$ and they uncouple respiration and decrease yeast growth rate as an artifact of overexpression ${ }^{24,25}$. To test whether the phospholipid fatty acyl composition changes seen with hUCP2 and hUCP3 overexpression were secondary to the uncoupling and growth inhibitory effects, we uncoupled yeast with dinitrophenol and measured the effect on mitochondrial phospholipid fatty acyl composition. Figure $4 \mathrm{a}$ shows that $0.11 \mathrm{mM}$ dinitrophenol in the culture medium inhibited growth of yeast containing empty vector to the same extent as did expression of UCP3, while $0.2 \mathrm{mM}$ dinitrophenol caused a greater growth defect. However, dinitrophenol did not affect the phospholipid fatty acyl composition of the mitochondria (Fig 4b), showing that it was not 


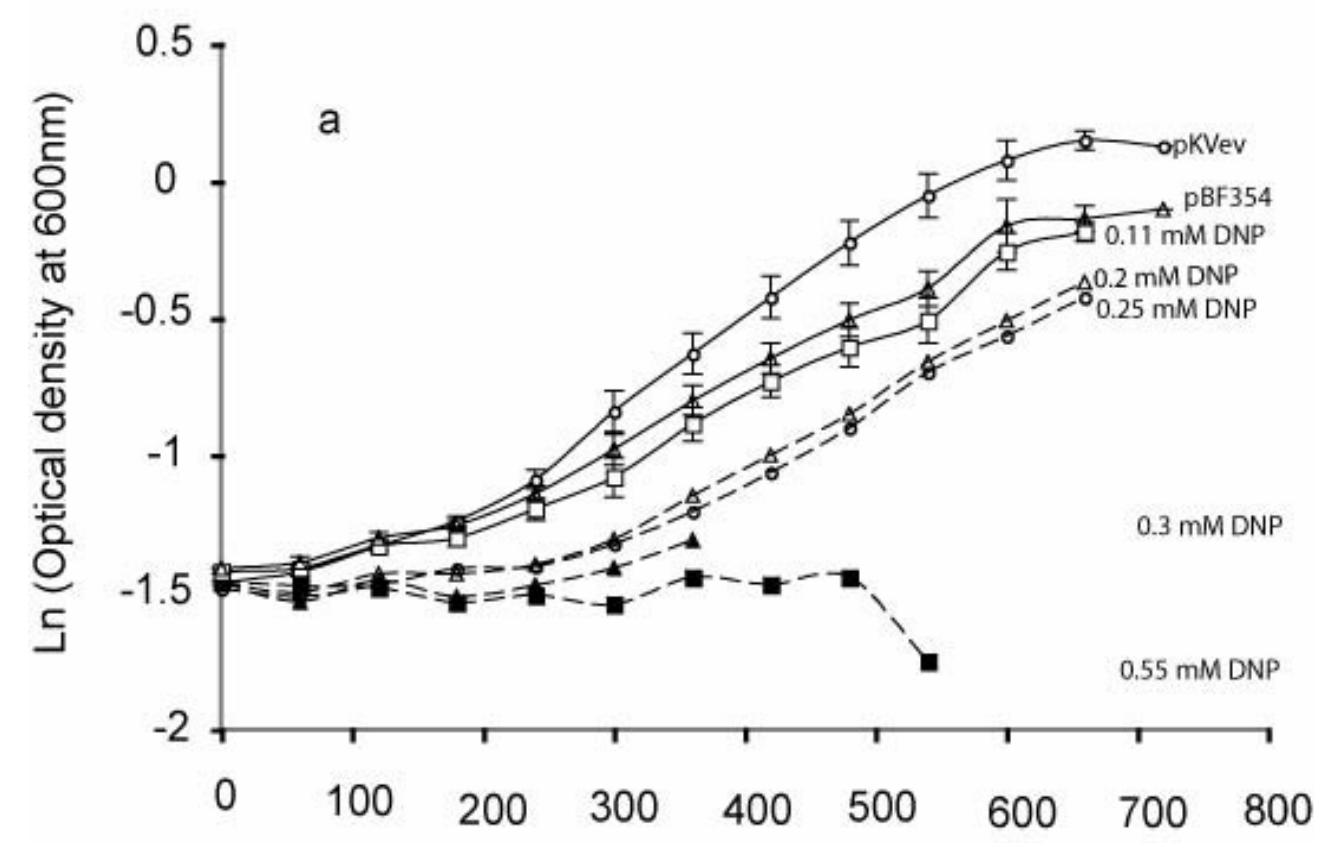

Time (min)

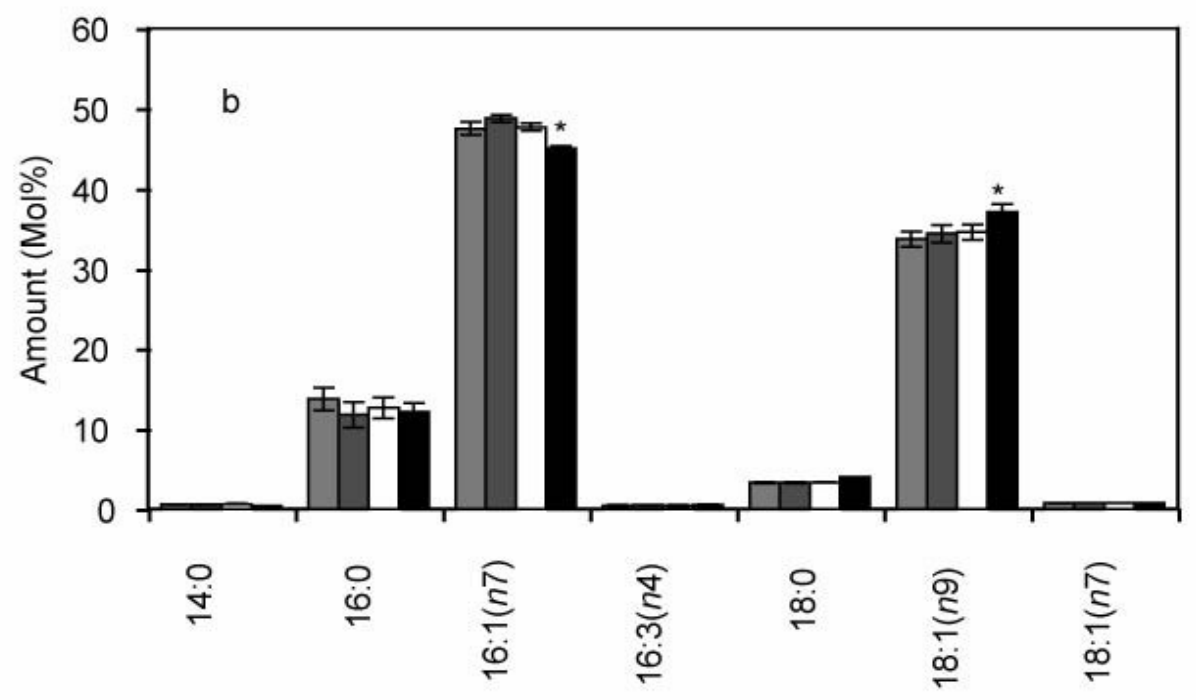

Fatty acyl group

Fig. 4. Comparison of the effects of uncoupling induced by hUCP3 and by dinitrophenol.

(a) Natural logarithmic growth curves of yeast expressing hUCP3 (pBF354) and UCP3 empty vector containing yeast (pKVev) grown with or without dinitrophenol.

$\longrightarrow$ pKVev; - $-0.55 \mathrm{mM}$ DNP;- $\rightarrow--0.3 \mathrm{mM}$ DNP;- $---0.25 \mathrm{mM}$ DNP;- $---0.2 \mathrm{mM}$ DNP

$\longrightarrow$ - $011 \mathrm{mM} \mathrm{DNP} \longrightarrow \mathrm{pBF} 354$

(b) Total mitochondrial phospholipid fatty acyl profile of mitochondria from yeast containing pKVev grown in $0.11 \mathrm{mM}$ dinitrophenol (light grey bars), pKVev grown in $0.22 \mathrm{mM}$ dinitrophenol (dark grey bars), pKVev (white bars) and yeast expressing hUCP3 (pBF354) (black bars). Values are means \pm SEM of 4 independent experiments. ${ }^{*} p<0.05$ compared to pKVev: $16: 1(n-7), p<0.0001 ; 18: 1(n-9), p=0.001$. 
just the uncoupling effect of UCP2 and UCP3 that caused the change in fatty acyl composition. The effect of UCP3 in Fig. 2a was seen again independently in Fig. 4b, confirming the earlier result. The lack of effect of dinitrophenol on $S$. cerevisiae mitochondrial phospholipid fatty acyl composition agrees with results showing no significant effect of growth rate on the phospholipid fatty acyl composition of Candida utilis $^{33}$.

\section{DISCUSSION}

The present study shows that heterologous high expression of non-functional hUCP2 and hUCP3 in yeast mitochondria had small but statistically significant effects on mitochondrial membrane phospholipid fatty acyl composition (Fig. 2), echoing the effects of expression of non-functional hUCP3 in mouse muscle ${ }^{23}$. However, the specific changes were different: in mouse 16:0, 18:1(n-9) and 18:2(n-6) decreased and 18:0 and 22:6(n-3) increased ${ }^{23}$, whereas in yeast 16:1(n-7) decreased and 18:1(n-9) increased (Fig. 2). This may be due to species differences since the fatty acyl profiles of mitochondrial phospholipids from the two organisms are very different, and the mechanisms that regulate composition may also differ. The effects on composition by UCP2 and 3 in the present studies and by UCP3 in previous studies $^{23}$ were not caused by (i) diet, since the different yeast strains were grown in identical media or (ii) high membrane protein content per $s e$, since high expression of mUCP1 (Fig 3b) did not give the same results as high expression of hUCP2 or hUCP3 (Fig 2). They were not secondary to (iii) increased uncoupling since dinitrophenol had no effect (Fig. 4). Heterologous expression in yeast of functional or non-functional mUCP1 did not lead to the same changes in fatty acyl profile, suggesting that the effects of non-functional hUCP2 and hUCP3 were relatively specific and were due to (iv) direct effects on fatty acyl composition of improperly folded hUCP2 and hUCP3.

Mouse mitochondria expressing hUCP3 have higher proton conductance than controls ${ }^{22}$ and the changes in fatty acyl profile match the general shift in fatty acyl composition in mitochondria with higher basal proton conductance that has been recorded across a wide range of different species and treatments. However, the changes in yeast mitochondria expressing hUCP2 and hUCP3 did not fit this general pattern: higher proton conductance in yeast expressing hUCP2 and hUCP3 $3^{24,26}$ correlated with higher 18:1(n-9) content (Figs. $2,3)$ rather than with lower 18:1(n-9) content as seen in the wider correlations. Thus there is no particular reason to suggest that the increased proton conductance of yeast mitochondria overexpressing non-functional hUCP2 or hUCP3 is caused by secondary changes in the membrane phospholipids rather than through the expressed proteins themselves.

UCP1 was the first member of the uncoupling protein family to be discovered ${ }^{13}$. The properties of UCP1 have been widely studied and well documented ${ }^{6}$ because UCP1 can be functionally expressed in bacteria, yeast or mammalian expression systems (heterologous expression). Ever since UCP2 and UCP3 were discovered $^{34}$, there has been no report in which heterologous expression of UCP2 or UCP3 in yeast, in mice, or in cultured cells, has led to an increase in proton conductance that has been demonstrated to be due to native UCP (protein) function.

All attempts to functionally expressed UCP2 and 3 heterologously, have been unsuccessful and characterised by artificial overexpression (protein content higher than physiological level) resulting in denatured protein most of which occur in extramitochondrial particles ${ }^{24-26,32}$.

The changes in fatty acid caused by UCP2 and 3 may be to minimise hydrophobic mismatch. In both mouse and yeast expressing hUCP3 there was a small but highly significant $(p<0.005)$ increase in the average chain length of the fatty acyl groups. This might minimise hydrophobic mismatch resulting from the improper insertion of the protein. In the 'mattress model' ${ }^{35,36}$, a membrane is visualised as a mattress of elastic medium (phospholipid) with localised springs (proteins). Insertion of an integral protein whose hydrophobic length is longer than the equilibrium hydrophobic thickness of its host lipid bilayer will result in hydrophobic mismatch and trigger a corrective increase in average fatty acyl chain length. In yeast, 16:1(n-7) (45 to $50 \mathrm{~mol} \%)$ and 18:1(n-9) 
(35\%) together make up more than $80 \%$ of the fatty acyl groups (Figs. 2-4), so the increase in average chain length may be most readily achieved by a small shift from $16: 1(n-7)$ to $18: 1(n-9)$, as observed. In mouse, the major fatty acyl groups are 16:0 (25\%), 18:0, 18:1(n-9), 18:2(n-6) and 22:6(n-3) (15-20\% each). Since an individual phospholipid in mammalian mitochondria usually has one saturated and one unsaturated fatty acyl group (to prevent segregation of the two types of acyl chain), the required increase in average chain length may be best achieved by small shifts from 16:0 to $18: 0$ and from 18:1(n-9) and 18:2(n-6) to $22: 6(n-3)$, as observed. This hypothesis explains the difference in the specific fatty acyl groups that are affected by hUCP 3 overexpression in mouse compared to yeast.

Overexpression of mUCP1 (figure $3 b$ ) was to mimic the effect of non-functional UCP2 and 3 but such overexpression is known to have some native UCP function ${ }^{25}$ indicating that the protein is not fully denatured. This could be the reason why such overexpression, caused no significant changes on phospholipid fatty acid composition.

In conclusion, expression of UCP2 or UCP3 in yeast caused an increase in 18:1(n-9) level and decrease in 16:1(n-7) level in yeast mitochondrial phospholipids.

\section{ACKNOWLEDGEMENT}

I thank the Cambridge Commonwealth and Overseas Trust, Gonville and Caius College, Cambridge and the Medical Research Council, UK for financial support.

\section{REFERENCES}

1. Kennedy, E. P. and Lehninger, A. L. (1949) Oxidation of fatty acids and tricarboxylic acid cycle intermediates by isolated rat liver mitochondria. J. Biol. Chem. 179: 957-972.

2. Mitchell, P. (1961) Uncoupling of phosphorylation to electron and hydrogen transfer by a chemiosmotic type of mechanism. Naturwissenschaften 191: 144-148.

3. Nicholls, D. G. (1974) Hamster brownadipose-tissue mitochondria. The control of respiration and the proton electrochemical potential gradient by possible physiological effectors of the proton conductance of the inner membrane. Eur. J. Biochem. 49: 573-583.

4. Nicholls, D. G. (1974) The influence of respiration and ATP hydrolysis on the proton-electrochemical gradient across the inner membrane of rat-liver mitochondria as determined by ion distribution. Eur. J. Biochem. 50: 305-315.

5. Brand, M. D. (1990) The proton leak across the mitochondrial inner membrane. Biochim. Biophys. Acta 1018: 128-133.

6. Nedergaard, J., Golozoubova, V., Matthias, A., Asadi, A., Jacobsson, A. and Cannon, B. (2001) UCP1: the only protein able to mediate adaptive nonshivering thermogenesis and metabolic inefficiency. Biochim. Biophys. Acta 1504: 82-106.

7. Green, K., Brand, M. D. and Murphy, M. P. (2004) Prevention of mitochondrial oxidative damage as a therapeutic strategy in diabetes. Diabetes 53 Suppl. 1: S110118.

8. Krauss, S., Zhang, C. Y., Scorrano, L., Dalgaard, L. T., St-Pierre, J., Grey, S. T. and Lowell, B. B. (2003) Superoxidemediated activation of uncoupling protein 2 causes pancreatic beta cell dysfunction. J. Clin. Invest. 112: 1831-1842.

9. Harper, J. A., Dickinson, K. and Brand, M. D. (2001) Mitochondrial uncoupling as a target for drug development for the treatment of obesity. Obes. Rev. 2: 255265.

10. Negre-Salvayre, A., Hirtz, C., Carrera, G., Cazenave, R., Troly, M., Salvayre, R., Penicaud, L. and Casteilla, L. (1997) A role for uncoupling protein-2 as a regulator of mitochondrial hydrogen peroxide generation. FASEB J. 11: 809815.

11. Argiles, J. M., Busquets, S. and LopezSoriano, F. J. (2002) The role of uncoupling proteins in pathophysiological states. Biochem. Biophys. Res. Commun. 293: $1145-1152$.

12. Nicholls, D. G. (1977) The effective proton conductance of the inner membrane of 
mitochondria from brown adipose tissue. Dependency on proton electrochemical potential gradient. Eur. J. Biochem. 77: 349-356.

13. Nicholls, D. G. (1977) Stoichiometries of proton translocation by mitochondria. Biochem. Soc. Trans. 5: 200-203.

14. Nicholls, D. G. and Rial, E. (1999) A history of the first uncoupling protein, UCP1. J. Bioenerg. Biomembr. 31: 399406.

15. Brookes, P. S., Buckingham, J. A., Tenreiro, A. M., Hulbert, A. J. and Brand, M. D. (1998) The proton permeability of the inner membrane of liver mitochondria from ectothermic and endothermic vertebrates and from obese rats: correlations with standard metabolic rate and phospholipid fatty acid composition. Comp. Biochem. Physiol. 119: B325-B334.

16. Brand, M. D., Steverding, D., Kadenbach, B., Stevenson, P. M. and Hafner, R. P. (1992) The mechanism of the increase in mitochondrial proton permeability induced by thyroid hormones. Eur. J. Biochem. 206: 775-781.

17. Brand, M. D., Turner, N., Ocloo, A., Else, P. L. and Hulbert, A. J. (2003) Proton conductance and fatty acyl composition of liver mitochondria correlates with body mass in birds. Biochem. J. 376: 741-748.

18. Hulbert, A. J. (2000) Thyroid hormones and their effects: a new perspective. Biol. Rev. Camb. Philos. Soc. 75: 519-631.

19. Hulbert, A. J., Else, P. L., Manolis, S. C. and Brand, M. D. (2002) Proton leak in hepatocytes and liver mitochondria from archosaurs (crocodiles) and allometric relationships for ectotherms. J. Comp. Physiol. 172: B387-B397.

20. Hulbert, A. J., Faulks, S., Buttemer, W. A. and Else, P. L. (2002) Acyl composition of muscle membranes varies with body size in birds. J. Exp. Biol. 205: 3561-3569.

21. Clapham, J. C., Arch, J. R., Chapman, H., Haynes, A., Lister, C., Moore, G. B., Piercy, V., Carter, S. A., Lehner, I., Smith, S. A., Beeley, L. J., Godden, R. J., Herrity, N., Skehel, M., Changani, K. K., Hockings, P. D., Reid, D. G.,
Squires, S. M., Hatcher, J., Trail, B., Latcham, J., Rastan, S., Harper, A. J., Cadenas, S., Buckingham, J. A., Brand, M. D. and Abuin, A. (2000) Mice overexpressing human uncoupling protein-3 in skeletal muscle are hyperphagic and lean. Nature 406: 415418.

22. Cadenas, S., Echtay, K. S., Harper, J. A., Jekabsons, M. B., Buckingham, J. A., Grau, E., Abuin, A., Chapman, H., Clapham, J. C. and Brand, M. D. (2002) The basal proton conductance of skeletal muscle mitochondria from transgenic mice overexpressing or lacking uncoupling protein-3. J. Biol. Chem. 277: 2773-2778.

23. Brand, M. D., Pamplona, R., PorteroOtin, M., Requena, J. R., Roebuck, S. J., Buckingham, J. A., Clapham, J. C. and Cadenas, S. (2002) Oxidative damage and phospholipid fatty acyl composition in skeletal muscle mitochondria from mice underexpressing or overexpressing uncoupling protein 3 . Biochem. J. 368: 597-603.

24. Harper, J. A., Stuart, J. A., Jekabsons, M. B., Roussel, D., Brindle, K. M., Dickinson, K., Jones, R. B. and Brand, M. D. (2002) Artifactual uncoupling by uncoupling protein 3 in yeast mitochondria at the concentrations found in mouse and rat skeletal-muscle mitochondria. Biochem. J. 361: 49-56.

25. Stuart, J. A., Harper, J. A., Brindle, K. M., Jekabsons, M. B. and Brand, M. D. (2001) A mitochondrial uncoupling artifact can be caused by expression of uncoupling protein 1 in yeast. Biochem. J. 356: 779-789.

26. Stuart, J. A., Harper, J. A., Brindle, K. M., Jekabsons, M. B. and Brand, M. D. (2001) Physiological levels of mammalian uncoupling protein 2 do not uncouple yeast mitochondria. J. Biol. Chem. 276: 18633-18639.

27. Roussel, D., Harding, M., Runswick, M. J., Walker, J. E. and Brand, M. D. (2002) Does any yeast mitochondrial carrier have a native uncoupling protein function? J. Bioenerg. Biomembr. 34: 165176. 
28. Brookes, P. S., Rolfe, D. F. and Brand, M. D. (1997) The proton permeability of liposomes made from mitochondrial inner membrane phospholipids: comparison with isolated mitochondria. J. Membr. Biol. 155: 167-174.

29. Daum, G. (1985) Lipids of mitochondria. Biochim. Biophys. Acta 822: 1-42.

30. Hoch, F. L. (1988) Lipids and thyroid hormones. Prog. Lipid Res. 27: 199-270.

31. Hoch, F. L. (1998) Cardiolipins and mitochondrial proton-selective leakage. $J$. Bioenerg. Biomembr. 30: 511-532.

32. Winkler, E., Heidkaemper, D., Klingenberg, M., Liu, Q. and Caskey, T. (2001) UCP3 expressed in yeast is primarily localized in extramitochondrial particles. Biochem. Biophys. Res. Commun. 282: 334-340.

33. McMurrough, I. and Rose, A. H. (1971) Effects of temperature variation on the fatty acid composition of Candida utilis. $J$. Bacteriol. 107: 753-758.

34. Ricquier, D. and Bouillaud, F. (2000) The uncoupling protein homologues: UCP1, UCP2, UCP3, StUCP and AtUCP. Biochem. J. 345 Pt 2: 161-179.

35. Mouritsen, O. G. and Bloom, M. (1984) Mattress model of lipid-protein interactions in membranes. Biophys. J. 46: 141-153.

36. Mouritsen, O. G. and Bloom, M. (1993) Models of lipid-protein interactions in membranes. Annu. Rev. Biophys. Biomol. Struct. 2: 145-171. 